\title{
Impact of lymphovascular invasion in bladder cancer Comparison of transurethral resection and total cystectomy: a retrospective cohort study
}

\section{Kei Yoneda}

Toho Daigaku Iryo Center Sakura Byoin

Naoto Kamiya ( $\square$ naoto.kamiya@med.toho-u.ac.jp )

Toho University Sakura Medical Center https://orcid.org/0000-0002-4183-2490

Takanobu Utsumi

Toho University Sakura Medical Center

\section{Ken Wakai}

Chiba Daigaku Daigakuin Igaku Kenkyuin Igakubu

\section{Ryo Oka}

Toho Daigaku Iryo Center Sakura Byoin

\section{Takumi Endo}

Toho Daigaku Iryo Center Sakura Byoin

\section{Masashi Yano}

Toho Daigaku Iryo Center Sakura Byoin

Nobuyuki Hiruta

Toho Daigaku Iryo Center Sakura Byoin

\section{Tomohiko Ichikawa}

Chiba Daigaku Daigakuin Igaku Kenkyuin Igakubu

Hiroyoshi Suzuki

Toho Daigaku Iryo Center Sakura Byoin

\section{Research article}

Keywords: bladder cancer, lymphovascular invasion, transurethral resection of bladder tumor, radical cystectomy

Posted Date: June 16th, 2020

DOI: https://doi.org/10.21203/rs.3.rs-29985/v1

License: (a) (i) This work is licensed under a Creative Commons Attribution 4.0 International License. Read Full License 


\section{Abstract}

Background: This study aimed to evaluate the associations of lymphovascular invasion (LVI) at first transurethral resection of bladder (TURBT) and radical cystectomy (RC) with survival outcomes, and to evaluate the concordance between LVI at first TURBT and RC.

Methods: We reviewed 216 patients who underwent first TURBT and 64 patients who underwent RC at our hospital. Medical records were reviewed uniformly, collecting the following data: age, sex, clinical and pathological T stage, neoadjuvant chemotherapy, grade, metastasis, urinary cytology, carcinoma in situ and LVI.

Results: LVI was identified in $22.7 \%$ of patients who underwent first TURBT, and $32.8 \%$ of patients who underwent RC. Univariate analysis identified $\geq c T 3$, metastasis and LVI at first TURBT as factors significantly associated with overall survival (OS) and cancer-specific survival (CSS). Multivariate analysis identified metastasis (hazard ratio $(H R) 6.560, p=0.009$ ) and LVI at first TURBT (HR 9.205, $p=0.003$ ) as significant predictors of CSS. On the other hand, in patients who underwent RC, $\geq p T 3$, inclusion of G3 and LVI were significantly associated with OS and CSS in univariate analysis. Multivariate analysis identified inclusion of G3 as a significant predictor of OS and CSS. The concordance rate between LVI at first TURBT and RC was $48.0 \%$. Patients with positive results for LVI at first TURBT and RC displayed poorer prognosis than other patients $(\mathrm{p}<0.05)$.

Conclusions: We found that the combination of LVI at first TURBT and RC was likely to provide a more significant prognostic factor.

\section{Background}

The presence of lymphovascular invasion (LVI) has been suggested to predict poor prognosis of bladder cancer, such as more advanced disease and recurrence, and has been reported as a poor prognostic factor even for other carcinomas [1-10]. As transurethral resection of bladder tumor (TURBT) is a standard treatment for bladder cancer, the utility of identifying LVI in specimens of TURBT has been suggested [1-7, 9-13]. The presence of LVI in TURBT specimens has been associated with pathologic upstaging and reduced recurrence-free survival (RFS) and progression-free survival [11]. Radical cystectomy (RC) is a standard treatment for muscle-invasive bladder cancer (MIBC), and the presence of LVI in RC specimens has also been associated with poor prognosis [14-17].

Further, LVI has been described as a risk factor in the guidelines of the American Urological Association. Those guidelines define high-grade and T1 tumors, recurrent high-grade and Ta tumors, high-grade Ta and large $(>3 \mathrm{~cm}$ ) tumor, multifocal high-grade Ta tumor, any carcinoma in situ (CIS), any Bacille de Calmette et Guérin failure in high-grade cases, any variant history, any high-grade prostatic urethral involvement, and any LVI as high-risk factors [18]. However, other guidelines such as those of the European Association of Urology do not define LVI as a high-risk factor [19]. Although T stage and grade are common high-risk factors, LVI has also been reported as independently associated with recurrence 
and progression, even when limited to pT1 high-grade patients [20]. We thus consider that LVI is also likely to represent an important high-risk factor in patients with other high-risk factors, such as T1 and high-grade bladder cancers. High-risk factors are important because the frequency of progression from non-MIBC (NMIBC) to MIBC is higher for high-risk patients than for low-risk patients. NMIBC progresses to MIBC in about $15 \%$ of patients, resulting in poor prognosis [21]. Although the standard treatment for MIBC is RC, up to $50 \%$ of patient treated in this manner experience disease recurrence and mortality [22]. For NMIBC patients, assessment of LVI at TURBT is very important for risk stratification and decision-making regarding further treatment. In comparison, for patients after RC, assessment of $\mathrm{LVI}$ at RC is suggested to help in determining adjuvant therapy.

As noted above, LVI has been studied in case reports of small numbers of cases, in larger international collaborative groups, and in meta-analyses. In particular, an extensive body of literature exists regarding LVI at RC, which is strongly suggested to be associated with poor clinical outcomes. On the other hand, LVI at TURBT has also been increasingly reported recently, and is suggested to be associated with poor prognosis. TURBT is the first-line treatment for bladder cancer, and we considered first-time (first) TURBT as the most important for diagnosis and treatment. Although many investigations have examined TURBT and RC individually, few have looked into LVI at TURBT and RC.

The present study aimed to evaluate the relationship between LVI on both TURBT and RC, as well as overall survival (OS) and cancer-specific survival (CSS). The present study also aimed to assess the concordance rate between TURBT and RC specimens with regard to the presence of LVI. Furthermore, we analyzed differences in prognosis due to the combination of LVI at TURBT and RC.

\section{Methods}

This was a retrospective study approved by the institutional review board (approval number: S17108). We identified 423 patients with bladder cancer treated by TURBT at Toho University Sakura Medical Center between January 2012 and December 2016. Moreover, a total of 291 patients underwent first TURBT for bladder cancer. Of those, 75 patients were excluded from the present study due to the following reasons: tumor of any other origin $(n=1)$; concurrent or recurrent bladder cancer with upper urinary tract urothelial carcinoma (UC) $(n=16)$; bladder Tis lesions only $(n=8)$; and missing values $(n=50)$. A final total of 216 patients were analyzed for prognostic factors of first TURBT, and to determine the utility of LVI (Supplementary table 1).

Furthermore, we analyzed prognostic factors for RC, and considered the relationship between LVI in samples from RC and LVI in samples from first TURBT. A total of 75 patients who underwent RC for bladder cancer were seen at our institution between 2010 and 2017. Of those, 11 patients were excluded for the following reasons: the pathology was not UC $(n=2)$; and missing values $(n=9)$. Eventually, a total of 64 patients were analyzed regarding prognostic factors for RC (Supplementary table 2). Twenty patients (31.3\%) received neoadjuvant chemotherapy (NAC). Generally, we administered two cycles of 
gemcitabine-cisplatin (GC). In addition, 50 patients underwent both first TURBT and RC at our institution, and we analyzed the concordance rate of each LVI. More details can be seen in the flow chart in Fig. 1 .

Medical records were surveyed retrospectively, and the following data were collected from medical charts: age at surgery, sex, clinical T stage, presence or absence of NAC, pathological T stage, grade (G1, G2, and G3), presence of metastasis, urinary cytology, CIS, and LVI. LVI was defined as the presence of tumor cells within an arterial, venous, or lymphatic lumen. LVI was defined as the presence of tumor cells within an arterial, venous, or lymphatic lumen. Presence of LVI in specimens was assessed using conventional hematoxylin and eosin (HE) staining. If we encountered any difficulty in evaluations using only HE staining, we added Elastica van Gieson staining. Positive urinary cytology was defined as $\geq$ class IV (malignancy suspected) according to Papanicolaou's classification. Lymph node metastasis and distant metastasis were collectively defined as metastasis. Pathological evaluation was performed by two of three pathologists as a council system in Toho University Sakura Medical Center using the tumor, node, metastasis classification system updated in 2009 and the new classification for grading non-invasive urothelial bladder carcinomas proposed by the World Health Organization and the International Society of Urological Pathology in 2004 [7-10].

\section{Statistical analysis}

Results are presented as median and range or mean \pm standard deviation, as appropriate. Continuous parametric variables were compared using $t$-tests. Non-parametric variables were compared using MannWhitney Utests. The Kaplan-Meier method was used to estimate OS and CSS following TURBT and RC, respectively. The log-rank test was used to compare statistical significances in each curve. After variables were selected in univariate analyses, regression analyses of Cox proportional hazards were performed to determine factors significantly associated with OS and CSS.

These statistical analyses were carried out using JMP Pro version 13 (SAS Institute, Cary, NC). All statistical tests were two-sided, and values of $p<0.05$ were considered significant.

\section{Results}

\section{LVI at first TURBT}

The clinical and pathological characteristics of patients who underwent first TURBT are shown in Supplementary table $1(n=216)$. Mean patient age was $71.3 \pm 9.3$ years, and males represented $79.6 \%$ of the study population. G3 was present in $43.5 \%$ of patients, $22.7 \%$ of patients had LVI, and only $9.7 \%$ had CIS. We compared the LVI-positive and -negative groups (Table 1). The LVI-positive group showed higher pathological T stage $(p<0.001)$ and greater frequency of G3 $(p<0.001)$ compared to the LVI-negative group. Subsequently, we analyzed prognostic factors from first TURBT (Table 2). Univariate analyses identified cT stage, metastasis and LVI as significantly associated with OS $(p<0.001, p<0.001, p=0.003$, respectively). Multivariate analysis identified metastasis (hazard ratio [HR] 4.717, 95\% confidence interval 
(Cl) $1.336-15.755 ; p=0.017$ ) as significant predictors of OS after first TURBT. According to univariate analyses, $\geq$ CT3 $(p<0.001)$, metastasis $(p<0.001)$ and LVI $(p<0.001)$ were significantly associated with CSS. Patients in the LVI-negative group at first TURBT $(n=167)$ displayed significantly better CSS than those in the LVI-positive group at first TURBT $(n=49 ; p<0.001)$ (Supplementary Fig. 1). Multivariate analysis identified metastasis (HR 6.560, 95\% Cl 1.593-29.027; $p=0.009)$ and LVI (HR 9.205, 95\% Cl $2.081-64.558 ; p=0.003$ ) as significant predictors of CSS.

\section{LVI at RC}

Clinical and pathological characteristics of patients who underwent RC are shown in Supplementary table $2(n=64)$. Mean patient age was $68.2 \pm 7.9$ years, and males represented $79.7 \%$ of the study population. G3 was present in $53.1 \%$ of patients, $32.8 \%$ of patients had LVI, and $10.9 \%$ had CIS. We analyzed the survival implications in 64 patients who underwent RC (Table 2). In univariate analyses, pT stage, tumor grade and LVI were significantly associated with OS $(p<0.001, p=0.001, p=0.001$, respectively). Multivariate analysis identified presence of $\mathrm{G} 3(\mathrm{HR} 7.942,95 \% \mathrm{Cl} 1.343-150.803 ; \mathrm{p}=0.020)$ as a significant predictor of OS. According to univariate analyses, $\geq c T 3$, presence of G3 and LVI were significantly associated with CSS $(p=0.002, p=0.003, p=0.001$, respectively). Patients in the LVInegative group at RC $(n=43)$ exhibited significantly better CSS than those in the LVI-positive group at RC $(n=21 ; p=0.001)$ (Supplementary Fig. 2). Multivariate analysis identified presence of G3 (HR 6.373, $95 \% \mathrm{Cl} 1.012-123.091 ; \mathrm{p}=0.048)$ as the only significant predictor of CSS.

\section{Comparison of LVI between first TURBT and RC}

We analyzed the concordance rate between LVI at first TURBT and LVI at RC (Table 3). We analyzed data from the 50 patients who underwent RC, excluding 14 patients who did not undergo first TURBT in our hospital or for whom data were missing. Of the 31 patients with LVI at first TURBT, 10 patients $(32.3 \%)$ also showed LVI at RC. Of the 19 patients without LVI at first TURBT, 14 patients (73.7\%) were also found to have no LVI at RC (Table 3).

We confirmed the effect of NAC on LVI in RC specimens. The clinical and pathological characteristics of patients according to use of NAC are shown in Supplementary table 3. In the group receiving NAC (NAC+ group) at first TURBT, $c$ T stage was higher $(p=0.006)$, and LVI was more frequently evident $(p=0.003)$ compared to the group not receiving NAC (NAC- group). On the other hand, patients in the NAC + group at RC displayed significantly higher pT stage than those in the NAC- group $(p=0.044)$. No significant difference in the presence or absence of LVI was evident between NAC + and NAC- groups at RC $(p=$ 0.312). In patients showing LVI ( $L V I+)$ at first TURBT, but not at RC, pT stage was pT0 in 9 patients (42.9\%). Supplementary table 4 shows changes in LVI according to the use of NAC in LVI + cases at first TURBT. LVI in RC specimens showed no significant difference according to use of NAC. No significant difference in LVI + or LVI- status was seen between the NAC + group $(n=14)$ and NAC- group $(n=17)$ in $L V I+$ cases at first TURBT $(p=0.252)$.

Finally, survival analysis was conducted across all combinations of LVI at first TURBT and RC. Patients with no evidence of LVI at both first TURBT and RC (TURBT-/RC-) had the best prognosis, and patients 
with evidence of LVI at both first TURBT and RC (TURBT+/RC+) had a worst prognosis $(p=0.016)$ (Fig. 2). Five-year CSS rates for each combination of LVI at first TURBT and RC were determined. Fiveyear CSS rate was $92.9 \%$ for patients with TURBT-/RC-, $80.0 \%$ for those with LVI at RC but not first TURBT (TURBT-/RC+), $77.0 \%$ for those with LVI at first TURBT but not at RC (TURBT+/RC-), and $50.0 \%$ for those with evidence of LVI at both first TURBT and RC (TURBT+/RC+).

\section{Discussion}

This study detected LVI in $22.7 \%$ of first TURBT specimens, and $32.8 \%$ of RC specimens. These rates were comparable to those described in the literature. The rate of LVI at TURBT has been reported as 6$70 \%$, and the rate of $\mathrm{LVI}$ at $\mathrm{RC}$ has been reported to range from $30-50 \%$ [1]. Clinical stage and pathological T stage were higher in the LVI-positive group than in the LVI-negative group at first TURBT. Moreover, we found that patients with LVI often showed evidence of G3 in the resected specimen. Patients with LVI were thus suggested to be more likely to be at high risk. In clinical practice, choosing the next treatment for patients with high-grade T1 is difficult. This is because high-risk patients are more likely to progress to MIBC, and RC is also a treatment option. Mathieu et al. showed that high-grade pT1 bladder cancer and LVI detected in biopsy specimens were poor prognostic factors for disease recurrence and progression [23]. RC is thus suggested to be a treatment option before progression for high-grade T1 patients with LVI.

At first TURBT, $\geq c T 3$, presence of metastasis, and LVI were found to be significantly associated with OS and CSS in univariate analyses. Furthermore, LVI was found to be a significant predictor of CSS in multivariate analysis. Various studies have reported that LVI was associated with CSS, but not with OS. A meta-analysis of LVI at first TURBT has shown that the pooled hazard ratio was significant for CSS (HR $1.35,95 \% \mathrm{Cl} 1.01-1.81 ; \mathrm{p}=0.04$ ), but not for OS (HR 1.55, 95\% Cl 0.90-2.67; $\mathrm{p}=0.11$ ) [11]. Moreover, Streeper et al. showed that patients with LVI of clinical stage I or II showed lower survival than those without LVI (HR 2.68, 95\% Cl 1.55-4.64; $p=0.049)$ [3]. Resnick et al. also associated LVI at TURBT with an increasing likelihood of node-positive disease (48.3\% vs. $25.0 \%$; $<<0.001)$ [24]. In addition, the association between LVI in TURBT specimens and RFS has also been reported, and LVI at TURBT is a poor prognostic factor $[6,20,25]$.

Subsequently, at RC, factors of $\geq \mathrm{pT} 3$, presence of $\mathrm{G} 3$ and presence of LVI were found to be significantly associated with OS and CSS in univariate analyses. In multivariate analysis, inclusion of G3 was identified as a significant predictor of OS and CSS. High tumor grade is included as a high-risk factor in all guidelines, and is also included as a factor in the nomogram that predicts risk of recurrence after RC [26]. On the other hand, LVI was shown to be significantly associated with OS and CSS in univariate analysis. Although LVI was not identified as a significant factor in multivariate analysis in the present study, several studies have reported LVI as associated with OS, CSS, and RFS even in multivariate analysis [16, 27-32]. Mari et al. summarized the relationship between LVI and clinical outcomes after RC [15]. They found that patients with LVI at RC exhibited higher risks of disease recurrence (HR $1.57,95 \% \mathrm{Cl}$ 1.45-1.70) and cancer-specific mortality (HR 1.59, 95\% Cl 1.48-1.73). In particular, LVI was associated 
with recurrence and cancer-specific mortality with lymph node-negative bladder cancer. We suggest that lymph node-negative patients with LVI were at increased risk of lymph node metastasis, and LVI was likely to be a risk factor for recurrence and cancer-specific mortality. In this study, 5 patients who underwent RC had lymph node metastasis, and positive LVI was seen at TURBT in all these patients. Lymph node metastasis was not a significant prognostic factor after RC, probably because the sample size was too small. However, metastasis should be considered a strong prognostic factor in general.

Next, we considered the concordance between LVI in first TURBT and RC specimens. A total of 10 patients (32.2\%) with LVI at first TURBT showed the presence of LVI at RC. On the other hand, a total of 14 patients $(73.7 \%)$ without LVI at first TURBT had negative LVI at RC. In other words, the concordance rate between LVI at TURBT and at RC was $48.0 \%$. The concordance rate was about $60-70 \%$ in some other reports, but was lower in this study $[3,24,33]$. The reasons for this were as follows. First, although many pathologists assess and diagnose specimens in daily clinical practice, a central pathological review of TURBT specimens was not performed in this study. Therefore, some variation in pathological evaluations may have been present. Second, some patients underwent NAC before RC at our hospital. Furthermore, the determination of which patients should undergo NAC was made at the discretion of each attending physician, and no clear, standardized criteria for selection were established. This might have affected the concordance rate. In fact, patients with LVI at first TURBT but not at RC (TURBT+, RC-) included patients who had undergone NAC and those whose pathological stage from the RC specimen was pT0. Moreover, we analyzed differences in prognosis due to the combination of LVI at first TURBT and RC, and found an interesting result: all patients with evidence of LVI at both first TURBT and RC displayed very poor prognosis and died within 2 years. LVI does not show a very high concordance rate, but may offer very useful information when both LVI at TURBT and RC are positive. Consequently, in patients with LVI at both first TURBT and RC, the results may be useful for considering adjuvant therapy after RC, such as adjuvant chemotherapy, to improve prognosis.

\section{Conclusions}

LVI at first TURBT and RC were associated with OS and CSS. Specifically, LVI at first TURBT was suggested to represent an independent prognostic factor for CSS. Furthermore, we found that the combination of LVI at first TURBT and RC was likely to offer a more significant prognostic factor than either factor individually.

\section{Abbreviations}

LVI

lymphovascular invasion

TURBT

transurethral resection of bladder tumor

$\mathrm{RC}$

radical cystectomy 
MIBC

muscle-invasive bladder cancer

CIS

carcinoma in situ

NMIBC

non-muscle invasive bladder cancer

OS

overall survival

CSS

cancer-specific survival

UC

urothelial carcinoma

NAC

neoadjuvant chemotherapy

GC

gemcitabine-cisplatin

HE

hematoxylin and eosin

HR

hazard ratio

\section{Declarations}

\section{Ethics approval and consent to participate}

This study was approved by the Toho University Sakura Medical Center Independent Ethics Committee. Ethics board approval number is $\mathrm{S} 17108$. Additional informed consent to participate was not required due to the retrospective nature of this study. However, we publish the details of this clinical study on our website, and we provide an opportunity for patients to reject (opt out).

\section{Consent for publication}

Not applicable.

\section{Availability of data and materials}

The datasets used and/or analyzed during the current study are available from the corresponding author on reasonable request. 


\section{Competing interests}

The authors declare that they have no competing interests.

\section{Funding}

This work was supported by a Grant-in-Aid from the Ministry of Education, Culture, Sports, Science and Technology (Contract grant number: 15k11030). The funders had no role in study design, data collection and analysis, decision to publish, or preparation of the manuscript.

\section{Authors' Contributions}

$\mathrm{KY}$ analyzed and interpreted the patient data, and was the major contributor in writing the manuscript. $\mathrm{KW}, \mathrm{RO}$ and TE participated in data acquisition and analysis. TU and MY participated in data acquisition and analysis. $\mathrm{NK}, \mathrm{TI}$ and $\mathrm{NH}$ also participated in the project design, supervised the project and participated in the writing and editing the manuscript. HS contributed to design of the work and supervised the project. All authors have read and approved the manuscript.

\section{Acknowledgements}

Not applicable.

\section{Authors' information}

\section{Affiliations}

Department of Urology, Toho University Sakura Medical Center, 564-1 Shimoshizu, Sakura-shi, Chiba, Japan

Kei Yoneda, Naoto Kamiya, Takanobu Utsumi, Ken Wakai, Ryo Oka, Takumi Endo, Masashi Yano, Hiroyoshi Suzuki

Department of Urology, Chiba University Graduate School of Medicine, 1-8-1 Inohana, Chuo-ku, Chiba-city, Chiba, Japan

Kei Yoneda, Ken Wakai, Tomohiko Ichikawa 
Department of Surgical Pathology, Toho University Sakura Medical Center, 564-1 Shimoshizu, Sakura-shi, Chiba, Japan

Nobuyuki Hiruta

\section{Corresponding author}

Correspondence to Naoto Kamiya.

\section{References}

1. Abufaraj M, Shariat SF, Foerster B, Pozo C, Moschini M, D'Andrea D, et al. Accuracy and prognostic value of variant histology and lymphovascular invasion at transurethral resection of bladder. World $\mathrm{J}$ Urol. 2018;36(2):231-40.

2. Matsuda T, Okuyama A. Incidence rate for bladder cancer in Japanese in Japan and in the United States from the Cancer Incidence in Five Continents. Jpn J Clin Oncol. 2017;47(1):284-5.

3. Streeper NM, Simons CM, Konety BR, Muirhead DM, Williams RD, O'Donnell MA, et al (2008) The significance of lymphovascular invasion in transurethral resection of bladder tumor and cystectomy specimens on the survival of patients with urothelial bladder cancer. BJU Int. 2008;103(4):475-9.

4. Andius P, Johansson SL, Holmäng S. Prognostic factors in stage T1 bladder cancer: tumor pattern (solid or papillary) and vascular invasion more important than depth of invasion. Urology. 2007;70(4):758-62.

5. Branchereau J, Larue S, Vayleux B, Karam G, Bouchot O, Rigaud J. Prognostic value of the lymphovascular invasion in high-grade stage pT1 bladder cancer. Clin Genitourin Cancer. 2013;11(2):182-8.

6. Cho KS, Seo HK, Joung JY, Park WS, Ro JY, Han KS, et al (2009) Lymphovascular invasion in transurethral resection specimens as predictor of progression and metastasis in patients with newly diagnosed T1 bladder urothelial cancer. J Urol. 2009;182(6):2625-30.

7. Ukai R, Hashimoto K, Nakayama H, Iwamoto T. Lymphovascular invasion predicts poor prognosis in high-grade pT1 bladder cancer patients who underwent transurethral resection in one piece. Jpn J Clin Oncol. 2017;47(5):447-52.

8. Yoneda K, Utsumi T, Somoto T, Wakai K, Oka R, Endo T, et al. External validation of two web-based postoperative nomograms predicting the probability of early biochemical recurrence after radical prostatectomy: a retrospective cohort study. Jpn J Clin Oncol. 2018;48(2):195-9.

9. Kang HW, Seo SP, Byun YJ, Piao XM, Kim YH, Jeong P, et al. Molecular progression risk score for prediction of muscle invasion in primary $\mathrm{T} 1$ high-grade bladder cancer. Clin Genitourin Cancer. 2018;16(4):274-80. 
10. Mari A, Kimura S, Foerster B, Abufaraj M, D'Andrea D, Hassler M, et al. A systematic review and metaanalysis of the impact of lymphvascular invasion in bladder cancer transurethral resection specimens. 2019;BJU Int 123(1):11-21.

11. Kim HS, Kim M, Jeong CW, Kwak C, Kim HH, Ku JH, et al. Presence of lymphovascular invasion in urothelial bladder cancer specimens after transurethral resections correlates with risk of upstaging and survival: A systematic review and meta-analysis. Urol Oncol. 2014;328:1191-9.

12. Wakai K, Utsumi T, Oka R, Endo T, Yano M, Kamijima S, et al. Clinical predictors for high-grade bladder cancer before first-time transurethral resection of the bladder tumor: a retrospective cohort study. Jpn J Clin Oncol. 2016;46(10):964-7.

13. Sha N, Xie L, Chen T, Xing C, Liu X, Zhang Y, et al. Impact of lymphovascular invasion on recurrence and progression rates in patients with pT1 urothelial carcinoma of bladder after transurethral resection. Onco Targets Ther. 2015;18(8):3401-6.

14. Martin JW, Vernez SL, Lotan Y, Abdelhalim A, Dutta R, Shokeir A, et al. Pathological characteristics and prognostic indicators of different histopathological types of urinary bladder cancer following radical cystectomy in a large single-center Egyptian cohort. World J Urol. 2018;36(11):1835-43.

15. Mari A, Kimura S, Foerster B, Abufaraj M, D'Andrea D, Gust KM, et al. A systematic review and metaanalysis of lymphovascular invasion in patients treated with radical cystectomy for bladder cancer. Urol Oncol. 2018;36(6):293-305.

16. Tilki D, Shariat SF, Lotan Y, Rink M, Karakiewicz PI, Schoenberg MP, et al. Lymphovascular invasion is independently associated with bladder cancer recurrence and survival in patients with final stage T1 disease and negative lymph nodes after radical cystectomy. BJU Int. 2013;111(8):1215-21.

17. Fritsche HM, Burger M, Svatek RS, Jeldres C, Karakiewicz PI, Novara G, et al. Characteristics and outcomes of patients with clinical T1 grade 3 urothelial carcinoma treated with radical cystectomy: results from an international cohort. Eur Urol. 2010;57(2):300-9.

18. Chang SS, Boorjian SA, Chou R, Clark PE, Daneshmand S, Konety BR, et al. Diagnosis and treatment of non-muscle invasive bladder cancer: AUA/SUO guideline. J Urol. 2016;196(4):1021-9.

19. Babjuk M, Böhle A, Burger M, Capoun O, Cohen D, Compérat EM, et al. EAU guidelines on non-muscleinvasive urothelial carcinoma of the bladder. Eur Urol. 2016;71(3):447-61.

20. Olsson H, Hultman P, Rosell J, Jahnson S. Population-based study on prognostic factors for recurrence and progression in primary stage T1 bladder tumours. Scand J Urol. 2013;47(3):188-95.

21. Woldu SL, Bagrodia A, Lotan Y. Guideline of guidelines: non-muscle-invasive bladder cancer. BJU Int. 2017;119(3):371-80.

22. Tian YF, Zhou H, Yu G, Wang J, Li H, Xia D, et al. Prognostic significance of lymphovascular invasion in bladder cancer after surgical resection: a meta-analysis. J Huazhong Univ Sci Technolog Med Sci. 2015;35(5):646-55.

23. Mathieu R, Lucca I, Rouprêt M, Briganti A, Shariat SF. The prognostic role of lymphovascular invasion in urothelial. Nat Rev Urol. 2016;13(8):471-9. 
24. Resnick MJ, Bergey M, Magerfleisch L, Tomaszewski JE, Malkowicz SB, Guzzo TJ. Longitudinal evaluation of the concordance and prognostic value of lymphovascular invasion in transurethral resection and radical cystectomy specimens. BJU Int. 2011;107(1):46-52.

25. Mitra AP, Skinner EC, Miranda G, Daneshmand S. A precystectomy decision model to predict pathological upstaging and oncological outcomes in clinical stage T2 bladder cancer. BJU Int. 2013;111(2):240-8.

26. Bochner $\mathrm{BH}$, Kattan MW, Vora KC. Postoperative nomogram predicting risk of recurrence after radical cystectomy for bladder cancer. J Clin Oncol. 2006;24(24):3967-72.

27. Park J, Park S, Song C, Doo C, Cho YM, Ahn H, et al. Effectiveness of adjuvant chemotherapy in transitional cell carcinoma of the urinary bladder with lymph node involvement and/or lymphovascular invasion treated by radical cystectomy. Urology. 2007;70(2):257-62.

28. Palmieri F, Brunocilla E, Bertaccini A, Guidi M, Pernetti R, Morselli-Labate AM, et al. Prognostic value of lymphovascular invasion in bladder cancer in patients treated with radical cystectomy. Anticancer Res. 2010;30(7):2973-6.

29. Bolenz C, Herrmann E, Bastian PJ, Michel MS, Wülfing C, Tiemann A, et al. Lymphovascular invasion is an independent predictor of oncological outcomes in patients with lymph node-negative urothelial bladder cancer treated by radical cystectomy: a multicentre validation trial. BJU Int. 2010;106(4):493-9.

30. Gondo T, Nakashima J, Ozu C, Ohno Y, Horiguchi Y, Namiki K, et al. Risk stratification of survival by lymphovascular invasion, pathological stage, and surgical margin in patients with bladder cancer treated with radical cystectomy. Int J Clin Oncol. 2012;17(5):456-61.

31. Shariat SF, Svatek RS, Tilki D, Skinner E, Karakiewicz PI, Capitanio U, et al. International validation of the prognostic value of lymphovascular invasion in patients treated with radical cystectomy. BJU Int. 2010;105(10):1402-12.

32. Muppa P, Gupta S, Frank I, Boorjian SA, Karnes RJ, Thompson RH, et al. Prognostic significance of lymphatic, vascular and perineural invasion for bladder cancer patients treated by radical cystectomy. Pathology. 2017;49(3):259-66.

33. Kunju LP, You L, Zhang Y, Daignault S, Montie JE, Lee CT. Lymphovascular invasion of urothelial cancer in matched transurethral bladder tumor resection and radical cystectomy specimens. J Urol. 2008;180(5):1928-32.

\section{Supplementary Figure Legends}

Supplementary fig. 1: Kaplan-Meier plot of cancer-specific survival divided into 2 groups according to LVI+ or LVI- at first TURBT

LVI: Iymphovascular invasion; TURBT: transurethral resection of bladder tumor 
Supplementary fig. 2: Kaplan-Meier plot of cancer-specific survival divided into 2 groups according to LVI+ or LVI- at RC

LVI: Iymphovascular invasion; RC: radical cystectomy

\section{Tables}


Table 1: Clinical and pathological characteristics stratified by LVI at first TURBT

\begin{tabular}{|c|c|c|c|}
\hline & LVI+ & LVI- & p-value \\
\hline & $\mathrm{n}=49$ & $\mathrm{n}=167$ & \\
\hline Age, years (range) & $74.0(43-89)$ & $71.0(40-92)$ & 0.30 \\
\hline Males, n (\%) & $38(78.6)$ & $134(80.2)$ & 0.73 \\
\hline Females, n (\%) & $11(22.4)$ & $33(19.8)$ & \\
\hline Clinical stage, n (\%) & & & $<0.001$ \\
\hline stage $0 \mathrm{a}, \mathrm{I}$ & $12(24.5)$ & $151(90.4)$ & \\
\hline stage II & $19(38.8)$ & $10(6.0)$ & \\
\hline stage III & 8 (16.3) & $2(1.2)$ & \\
\hline stage IV & $10(20.4)$ & $4(2.4)$ & \\
\hline Pathological T stage, n (\%) & & & $<0.001$ \\
\hline pTa & $2(4.0)$ & $98(58.7)$ & \\
\hline pT1 & $15(30.0)$ & $61(36.5)$ & \\
\hline$\square$ pT2 & $32(64.0)$ & $8(4.8)$ & \\
\hline Inclusion of $\mathrm{G} 3, \mathrm{n}(\%)$ & $38(75.5)$ & $56(33.5)$ & $<0.001$ \\
\hline CIS, n (\%) & $6(12.2)$ & $15(9.0)$ & 0.53 \\
\hline
\end{tabular}

LVI: lymphovascular invasion; TURBT: transurethral resection of bladder tumor; CIS: carcinoma in situ 
Table 2: Uni- and multivariate analyses of clinicopathological variables for OS and CSS after each of first TURBT and $\mathrm{RC}$ 


\section{Patients who underwent first TURBT $(n=216)$}

\begin{tabular}{|c|c|c|c|c|}
\hline & \multicolumn{2}{|c|}{ OS } & \multicolumn{2}{|r|}{ CSS } \\
\hline & Univariate & Multivariate & Univariate & Multivariate \\
\hline & p-value & HR (95\%CI) & $\mathrm{p}$-value & HR (95\%CI) \\
\hline & & p-value & & p-value \\
\hline \multirow[t]{3}{*}{ cT stage (<3 / प3) } & $<0.001$ & 2.2917 & $<0.001$ & 2.4901 \\
\hline & & $0.6861-7.0965$ & & $0.6045-10.8688$ \\
\hline & & 0.173 & & 0.21 \\
\hline \multirow[t]{3}{*}{ Metastasis $(-/+)$} & $<0.001$ & 4.7173 & $<0.001$ & 6.5595 \\
\hline & & $1.3359-15.755$ & & $1.5928-29.0273$ \\
\hline & & 0.017 & & 0.009 \\
\hline Grade (G1, 2 / G3) & 0.61 & & 0.16 & \\
\hline \multirow[t]{3}{*}{ LVI $(-/+)$} & 0.003 & 1.9662 & $<0.001$ & 9.20544 \\
\hline & & $0.7860-4.7265$ & & $2.0813-64.5576$ \\
\hline & & 0.145 & & 0.003 \\
\hline CIS $(-/+)$ & 0.23 & & 0.36 & \\
\hline Urinary cytology $(-/+)$ & 0.91 & & 0.88 & \\
\hline
\end{tabular}

\section{Patients who underwent RC ( $\mathrm{n}=64)$}

\begin{tabular}{|c|c|c|c|c|}
\hline & \multicolumn{2}{|c|}{ OS } & \multicolumn{2}{|c|}{ CSS } \\
\hline & Univariate & Multivariate & Univariate & Multivariate \\
\hline & $\mathrm{p}$-value & HR (95\%CI) & $\mathrm{p}$-value & HR (95\%CI) \\
\hline & & p-value & & p-value \\
\hline pT stage $(<3 /$ / 3$)$ & $<0.001$ & 1.8240 & 0.002 & 1.7037 \\
\hline
\end{tabular}




\begin{tabular}{|c|c|c|c|c|}
\hline & & 0.34 & & 0.42 \\
\hline Metastasis $(-/+)$ & 0.49 & & 0.75 & \\
\hline NAC $(-/+)$ & 0.71 & & 0.91 & \\
\hline Grade (G1, 2 / G3) & 0.001 & 7.9418 & 0.003 & 6.3727 \\
\hline
\end{tabular}

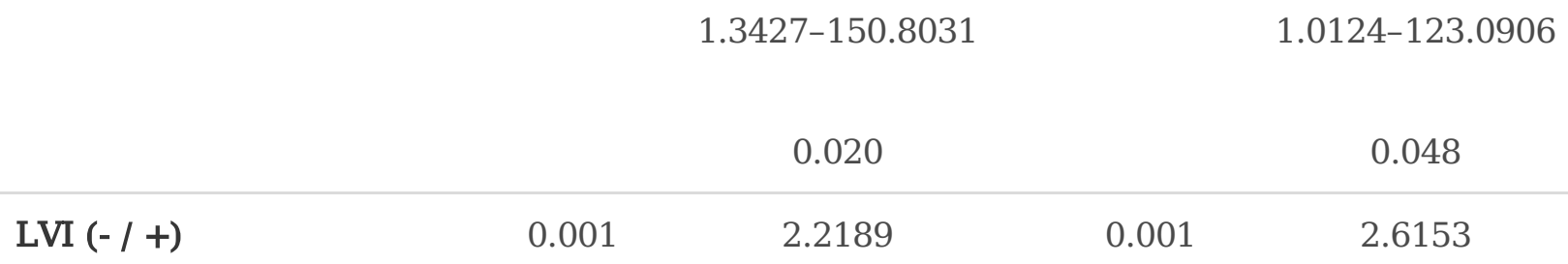

$0.6237-8.4612$

$0.6663-11.4548$

0.22

0.17

$\begin{array}{lll}\text { CIS }(-/+) & 0.63 & 0.74\end{array}$

OS: overall survival; CSS: cancer-specific survival; TURBT: transurethral resection of bladder tumor; RC: radical cystectomy; HR: hazard ratio; NAC: neoadjuvant chemotherapy; LVI: lymphovascular invasion; CIS: carcinoma in situ 
Table 3: Concordance between LVI at first TURBT and RC

\begin{tabular}{l|c|c|c}
\hline & LVI+, TURBT & LVI-, TURBT & Total \\
& $\mathbf{n}(\%)$ & $\mathbf{n}(\%)$ & \\
\hline LVI, RC & $10(20 \%)$ & $5(10 \%)$ & 15 \\
\hline LVI-, RC & $21(42 \%)$ & $14(28 \%)$ & 35 \\
\hline Total & 31 & 19 & \\
\hline
\end{tabular}

LVI: lymphovascular invasion; TURBT: transurethral resection of bladder tumor; RC: radical cystectomy

\section{Figures}
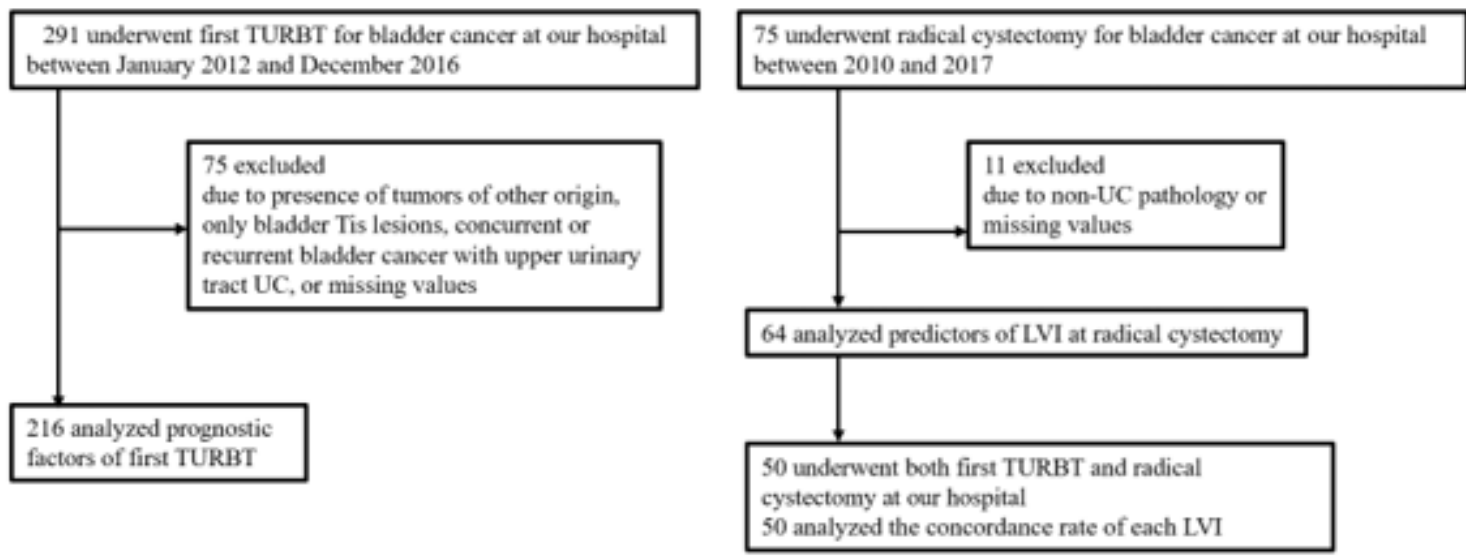

\section{Figure 1}

Flow chart for patient selection. TURBT: transurethral resection of bladder tumor; UC: urothelial carcinoma; LVI: lymphovascular invasion 


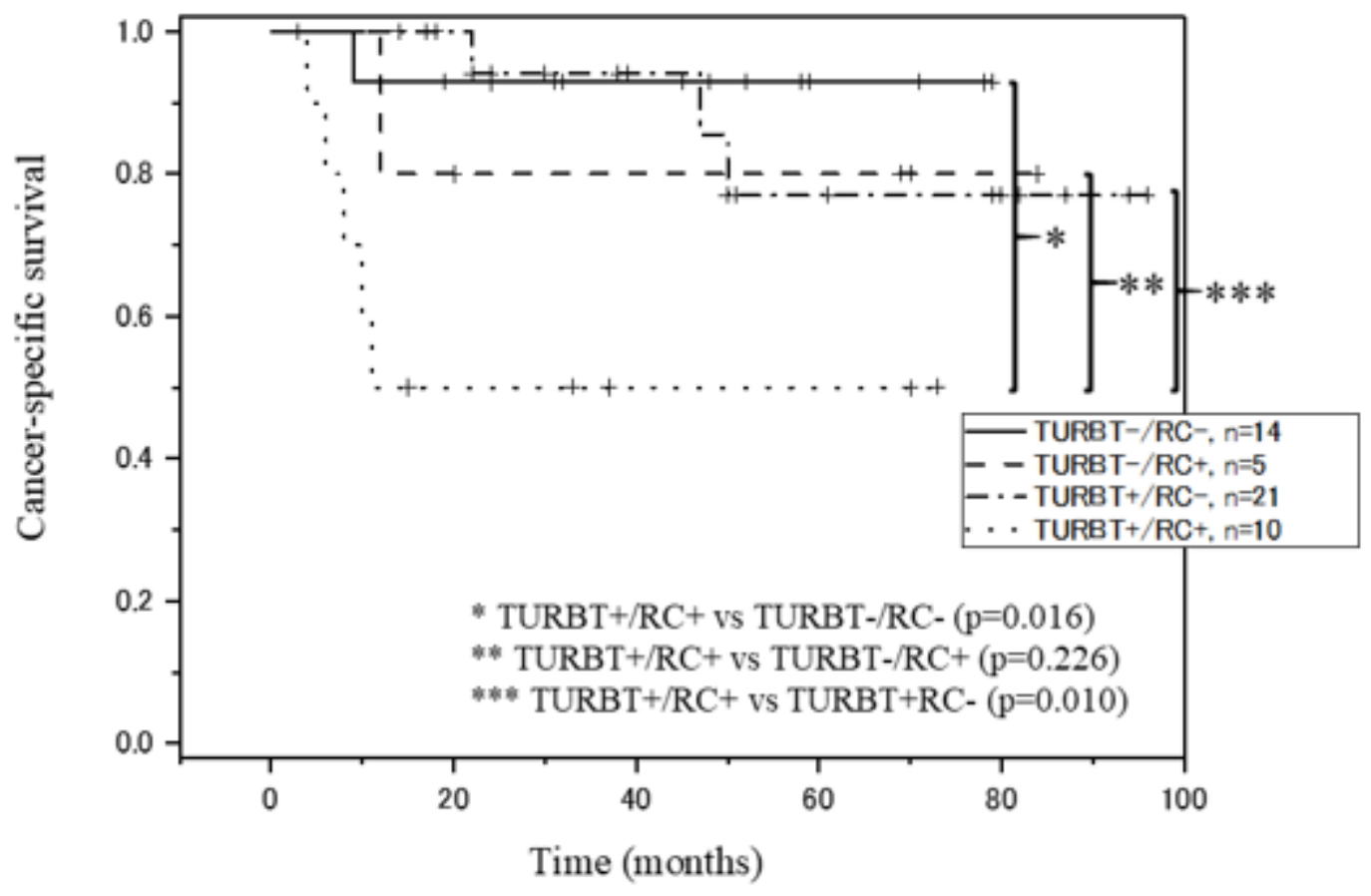

Figure 2

Cancer-specific survival in all combinations of LVI at first TURBT and RC. LVl: lymphovascular invasion; TURBT: transurethral resection of bladder tumor; RC: radical cystectomy

\section{Supplementary Files}

This is a list of supplementary files associated with this preprint. Click to download.

- LVIYonedaSupplementaryTables.docx

- LVIYonedaSupplementaryFigures2020.5.13ver2.docx 